\title{
Erratum to: Microscopic visualization of testosterone in mouse testis by use of imaging mass spectrometry
}

\author{
Shuichi Shimma ${ }^{1}$ - Henri-Obadja Kumada ${ }^{2}$ Hisanori Taniguchi ${ }^{2,3}$ • Alu Konno ${ }^{4,5}$. \\ Ikuko Yao $^{2,4,5} \cdot$ Kyoji Furuta $^{6}$ - Tadashi Matsuda ${ }^{3}$ - Seiji Ito ${ }^{2}$
}

Received: 10 August 2016/Accepted: 10 August 2016/Published online: 10 September 2016

(C) Springer-Verlag Berlin Heidelberg 2016

\section{Erratum to: Anal Bioanal Chem}

DOI 10.1007/s00216-016-9594-9

The authors would like to call the reader's attention to the fact that in the original version of this article unfortunately a reference was wrongly numbered. In the section "Material and methods" the first sentence in the paragraph "On-tissue derivatization with GT" should read: "We optimized the on-tissue derivatization method using GT based on a previous report [6]".

Published in the topical collection New Applications of Mass Spectrometry in Biomedicine with guest editors Mitsutoshi Setou and Toshimitsu Niwa.

Shuichi Shimma and Henri-Obadja Kumada contributed equally to this work.

The online version of the original article can be found at 10.1007/s00216016-9594-9.

Seiji Ito

ito@hirakata.kmu.ac.jp

1 Division of Advanced Science and Biotechnology, Graduate School of Engineering of Osaka University, 2-1 Yamadaoka,

Suita, Osaka 565-0871, Japan

2 Department of Medical Chemistry, Kansai Medical University, 2-5-1 Shin-machi, Hirakata, Osaka 573-1010, Japan

3 Department of Urology and Andrology, Kansai Medical University, 2-5-1 Shin-machi, Hirakata, Osaka 573-1010, Japan
4 Department of Optical Imaging, Institute for Medical Photonics Research, Preeminent Medical Photonics Education \& Research Center, Hamamatsu University School of Medicine, 1-20-1 Handayama, Higashi-ku, Hamamatsu, Shizuoka 431-3192, Japan

5 JST, ERATO, Sato Project, Tokyo 160-8582, Japan

6 Regeneration and Advanced Medical Science, Gifu University Graduate School of Medicine, Yanagido 1-1, Gifu, Gifu 501-1193, Japan 